Journal of Chromatography A

October 2015, Volume 1416, Pages 10-21

http://dx.doi.org/10.1016/i.chroma.2015.08.064

http://archimer.ifremer.fr/doc/00277/38848/

(c) 2015 Elsevier B.V. All rights reserved.

\title{
High resolution mass spectrometry for quantitative analysis and untargeted screening of algal toxins in mussels and passive samplers
}

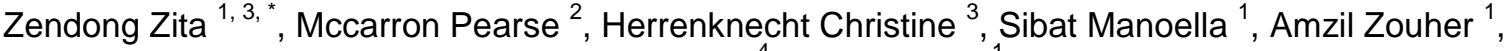 \\ Cole Richard B. ${ }^{4}$, Hess Philipp ${ }^{1}$
}

${ }^{1}$ Ifremer, Laboratoire Phycotoxines, Rue de l'lle d'Yeu, 44311 Nantes, France

${ }^{2}$ National Research Council of Canada, Biotoxin Metrology, Measurement Science and Standards, 1411 Oxford St, Halifax, Nova Scotia B3H $3 Z$ 1, Canada

${ }^{3}$ LUNAM, Université de Nantes, MMS EA2160, Faculté de Pharmacie, 9 rue Bias, 44035 Nantes, France

${ }^{4}$ Institut Parisien de Chimie Moléculaire, UMR 8232, Université Pierre et Marie Curie (Paris VI), 4 Place Jussieu, 75252 Paris, France

* Corresponding author : Zita Zendong, email address : Zita.Zendong@ifremer.fr

\begin{abstract}
:
Measurement of marine algal toxins has traditionally focussed on shellfish monitoring while, over the last decade, passive sampling has been introduced as a complementary tool for exploratory studies. Since 2011, liquid chromatography-tandem mass spectrometry (LC-MS/MS) has been adopted as the EU reference method (No. 15/2011) for detection and quantitation of lipophilic toxins. Traditional LC-MS approaches have been based on low-resolution mass spectrometry (LRMS), however, advances in instrument platforms have led to a heightened interest in the use of high-resolution mass spectrometry (HRMS) for toxin detection. This work describes the use of HRMS in combination with passive sampling as a progressive approach to marine algal toxin surveys. Experiments focused on comparison of LRMS and HRMS for determination of a broad range of toxins in shellfish and passive samplers.
\end{abstract}

Matrix effects are an important issue to address in LC-MS; therefore, this phenomenon was evaluated for mussels (Mytilus galloprovincialis) and passive samplers using LRMS (triple quadrupole) and HRMS (quadrupole time-of-flight and Orbitrap) instruments. Matrix-matched calibration solutions containing okadaic acid and dinophysistoxins, pectenotoxin, azaspiracids, yessotoxins, domoic acid, pinnatoxins, gymnodimine A and 13-desmethyl spirolide C were prepared. Similar matrix effects were observed on all instruments types. Most notably, there was ion enhancement for pectenotoxins, okadaic acid/dinophysistoxins on one hand, and ion suppression for yessotoxins on the other. Interestingly, the ion selected for quantitation of PTX2 also influenced the magnitude of matrix effects, with the sodium adduct typically exhibiting less susceptibility to matrix effects than the ammonium adduct. As expected, mussel as a biological matrix, quantitatively produced significantly more matrix effects than passive sampler extracts, irrespective of toxin. Sample dilution was demonstrated as an effective measure to reduce matrix effects for all compounds, and was found to be particularly useful for the non-targeted 
approach.

Limits of detection and method accuracy were comparable between the systems tested, demonstrating the applicability of HRMS as an effective tool for screening and quantitative analysis. HRMS offers the advantage of untargeted analysis, meaning that datasets can be retrospectively analyzed. HRMS (full scan) chromatograms of passive samplers yielded significantly less complex data sets than mussels, and were thus more easily screened for unknowns. Consequently, we recommend the use of HRMS in combination with passive sampling for studies investigating emerging or hitherto uncharacterized toxins.

\section{Highlights}

Quantitative HRMS-method developed for targeted screening of biotoxins. Advantage of HRMS over LRMS with regards to untargeted screening of unknowns. Similar magnitude and direction of matrix effects in HRMS compared to LRMS. Less matrix effects with passive sampler matrix compared to mussel matrix.

Keywords : Monitoring, Marine toxins, Passive sampling, SPATT, Matrix effects 


\section{INTRODUCTION}

A number of micro-algae produce marine toxins that can be accumulated in filter-feeding shellfish species such as mussels and oysters, and thus lead to human intoxication through consumption [1]. For several decades, the complexity of the toxins produced by these algae has impeded method development due to the lack of reference calibrants and materials. Therefore, generic mouse bioassays were often used, despite commonly accepted drawbacks [2]. Liquid chromatography coupled to tandem mass spectrometry (LC-MS/MS) has become a versatile tool for the analysis of food and environmental contaminants, including toxins. LC-MS/MS is now the reference method for the detection and quantitation of toxins produced by harmful algae [3]. To achieve this goal, different studies have developed and validated quantitative methods for the analysis of phycotoxins, typically using low resolution mass spectrometry (LRMS) [4-9]. This technique is now being increasingly used for monitoring $[10,11]$ and for characterization of reference materials [12, 13]. Additionally, methods using high resolution mass spectrometry (HRMS) have recently been developed and quantitatively validated for some marine toxins [14-16].

However, an important issue to address when developing or validating a quantitative analytical method using LC-MS via electrospray (ESI) and atmospheric pressure ionization (API) sources is the possible occurrence of matrix effects $[17,18]$. Matrix effects are considered to be an alteration in analyte response due to the presence of co-eluting compounds, either due to mass interference (isobaric compounds) or alteration of the desorption/ionization efficiency due to co-elution. These co-eluting compounds may increase (ion enhancement) or reduce (ion suppression) the desorption/ionization of the targeted analyte [19, 20]. Matrix effects may arise from different co-eluting components: endogenous compounds already present as sample constituents and still present after extraction or sample pre-treatment, or from reagents added to the mobile phase to improve chromatographic separation and peak shape [21], as well as from interfering materials used during extraction procedures or even from variable elution flow-rates [22]. Matrix effects can be easily detected when comparing the response obtained from standard solutions to those from spiked matrix extracts. In the presence of matrix effects, both identification and determination of analytes can be affected [22]. Therefore, the evaluation of matrix effects in MS detection and solutions to overcome them should be examined in the early stages of development of new methods. Several approaches have been used to alleviate matrix effects in the quantitative analysis of lipophilic marine toxins. These approaches include SPE cleanup and column flushing [23, 24], matrix-matched calibration and standard addition [24-26], reduction of the injection volume [11], use of an internal standard and use of a different ionization source such as APCI [19].

For applications that require analyses of complex biological samples, the use of HRMS can offer at least two major advantages: (i) the ability to overcome mass interferences stemming from overlapping signals of isobaric species (at low resolution such interferences lead to overestimation of the quantity of the analyte present) and (ii) non-targeted screening (where mass spectrometry is used to survey the contents of a complex mixture). In the field of toxins a good example of HRMS dealing with interfering isobaric compounds is the case of anatoxin-a, which may be hampered by the presence of phenylalanine [27]. HRMS has also been the prime technique for non-targeted screening of complex samples for unknowns, employing Orbitrap and Time-of-Flight mass spectrometers [9, 28, 29].

While monitoring of biotoxins has traditionally been carried out in mussels, passive samplers, also referred to as Solid Phase Adsorption Toxin Tracking (SPATT) have been more recently introduced to detect toxins in the marine environment [30]. Subsequently, many studies have successfully implemented passive sampling, using mainly the HP20 resin, to detect lipophilic toxins in different aquatic environments [31-35]. This technique has not yet proven to be useful as a monitoring tool for early warning of harmful algal blooms [36]. However, passive samplers have the advantage that unlike 
in mussels, the adsorbed toxins do not undergo biotransformation. Mussels have traditionally been used in many monitoring programs since they can be classified as a sentinel species due to the relatively unselective feeding of mussels compared to other bivalve mollusks, e.g. oysters.

In this study, we evaluate and compare matrix effects caused by mussel matrix and passive sampler components in the analysis of different phycotoxins, using both low and high resolution mass spectrometers. As a complement to the overall non-targeted approach employing HRMS, a range of toxins was investigated quantitatively: from relatively hydrophilic toxins such as domoic acid (DA) and yessotoxins (YTX and homo-YTX), over toxins of intermediate lipophilicity such as pinnatoxins E, F and G (PnTX-E, -F, -G), gymnodimine A (GYM-A), 13-desmethylspirolide-C (13-desmeSPX-C), to the more lipophilic ones including azaspiracids 1 to 3 (AZA1, -2, -3), okadaic acid (OA) dinophysistoxins 1 and 2 (DTX1, -2), pectenotoxin 2 (PTX2) and brevetoxin-1 and 2 (BTX1, -2). A chromatographic separation method was developed and optimized to obtain good separation of the toxins of interest. Matrix matched calibration curves, prepared using mussel and passive sampler extracts, were injected on different analytical systems with low resolution (triple quadrupole) and high resolution (orbitrap and quadrupole time-of-flight) mass spectrometers. The impact of the ion selected for quantitation, sample dilution and use of low or high resolution detectors on matrix effects were assessed. Finally, the study evaluated the benefits of passive sampler matrix as a complementary tool to traditionally used shellfish matrix (mussels) with the help of HRMS for an untargeted, exploratory approach.

\section{EXPERIMENTAL}

\subsection{Chemicals and reagents}

Certified calibration solutions were from the National Research Council of Canada (NRCC, Halifax, NS, Canada). These included calibration solution CRMs: domoic acid (DA), azaspiracids 1, 2 and 3 (AZA1-3), pectenotoxin 2 (PTX2), okadaic acid (OA) dinophysistoxins 1 and 2 (DTX1 and -2), yessotoxin (YTX), homo-yessotoxin (homo-YTX), 13-desmethyl spirolide C (13-desmeSPX-C), pinnatoxin G (PnTX-G) and gymnodimine A (GYM-A); and mussel tissue CRMs: CRM-ASP-Mus-d, CRM-DSP-Mus-c and CRM-AZA-Mus. A multitoxin tissue material CRM-FDMT-1 undergoing certification, well-characterized in-house calibration solutions for PnTX-E and F, brevetoxins 1 and 2 (BTX1 and -2), 20-methyl spirolide G (20-me-SPX-G) and pectenotoxin-2-seco acid (PTX2sa), as well as a mussel extract (Bruckless, Donegal, Ireland - 2005) containing different azaspiracids were also provided by NRC.

Alexandrium ostenfeldii (A. ostenfeldii) extract containing 13,19-didesmethyl spirolide C (13,19didesme-SPX-C) and Ostreopsis ovata (O. ovata) extract containing ovatoxin a (OvTX-a) were obtained from Ifremer as previously described $[33,37]$. Those extracts were mixed with some of the abovementioned certified and in-house reference toxin calibration solutions as well as the mussel extract from Bruckless to obtain a composite multi-toxin sample, used for optimization of chromatographic separation.

HPLC-grade methanol, acetonitrile and formic acid (98\%) were obtained from Sigma Aldrich (Steinheim, Germany) and Caledon (Georgetown, ON, Canada). Ammonium formate was from Fluka (St. Louis, MI, USA). Milli-Q water was produced in-house at $18 \mathrm{M} \Omega / \mathrm{cm}$ quality, using a Milli-Q integral 3 system (Millipore). For analyses with HRMS instruments, acetonitrile and water of LCMSgrade were obtained from Fisher Scientific (Illkirch, France). 


\subsection{Instrumentation and analytical methods}

147

148

149

150

151

152

153

154

155

156

157

158

\subsubsection{LC-MS/MS systems}

System A: Triple quadrupole $(Q q Q)$ :

An Agilent HPLC 1100 series system (1.58 min dwell time) was connected to an API4000 ${ }^{\mathrm{TM}}$ mass spectrometer (AB Sciex) equipped with a TurboIonSpray ${ }^{\mathrm{TM}}$ ionization source. For quantitation, the mass spectrometer was operated in MRM mode, scanning two transitions for each toxin. Q1 and Q3 resolutions of the instrument were set at Unit (arbitrary terms). Data were acquired in scheduled MRM and the target scan time was $1 \mathrm{~s}$ in both positive and negative modes. MRM detection windows were set at $45 \mathrm{~s}$ in both polarities. Data acquisition was carried out with Analyst 1.6 Software (AB Sciex). Optimized parameters are shown in Table 1.

Table 1: Optimized transitions selected for scheduled MRM method.

\begin{tabular}{|c|c|c|c|c|c|c|}
\hline Toxin & DP [V] & Q1 & $\begin{array}{c}\text { Q3 } \\
\text { quantifier }\end{array}$ & $\mathrm{CE}[\mathrm{eV}]$ & $\begin{array}{c}\text { Q3 } \\
\text { qualifier }\end{array}$ & $\mathrm{CE}[\mathrm{eV}]$ \\
\hline$\overline{D A}$ & 61 & 312.1 & 266.1 & 23 & 161.1 & 35 \\
\hline$O v T X-a$ & 65 & 1315.7 & 327.1 & 45 & 1298 & 25 \\
\hline GYM-A & 90 & 508.4 & 490.2 & 30 & 392.3 & 50 \\
\hline 13,19-didesMe-C & 120 & 678.5 & 430.5 & 45 & 164.5 & 65 \\
\hline 13-desmeSPX-C & 90 & 692.5 & 164.1 & 70 & 444.2 & 60 \\
\hline 20-me-SPX-G & 85 & 706.6 & 164.1 & 70 & 346.3 & 50 \\
\hline PnTX-G & 125 & 694.5 & 164.1 & 80 & 458.3 & 60 \\
\hline PnTX-E & 125 & 784.5 & 164.1 & 80 & 766.5 & 60 \\
\hline$P n T X-F$ & 125 & 766.5 & 164.1 & 80 & 748.5 & 60 \\
\hline$A Z A 1$ & 60 & 842.5 & 672.4 & 65 & 362.3 & 75 \\
\hline$A Z A 2$ & 60 & 856.5 & 672.4 & 65 & 362.3 & 75 \\
\hline$A Z A 3$ & 60 & 828.5 & 658.4 & 65 & 362.3 & 75 \\
\hline AZA6 & 110 & 842.5 & 658.4 & 65 & 362.3 & 75 \\
\hline$A Z A 33$ & 110 & 716.5 & 698.5 & 40 & 362.4 & 70 \\
\hline AZA34 & 116 & 816.5 & 798.4 & 41 & 672.5 & 69 \\
\hline BTX1 & 70 & 884.6 & 221.1 & 35 & 403.4 & 30 \\
\hline BTX2 & 90 & 912.5 & 895.5 & 19 & 877.5 & 29 \\
\hline PTX2 & 80 & 876.5 & 823.5 & 35 & 213.1 & 55 \\
\hline PTX2sa & 85 & 894.5 & 823.5 & 35 & 213.1 & 60 \\
\hline$O A, D T X 2$ & -80 & 803.5 & 255.1 & -65 & 113.1 & -85 \\
\hline$D T X 1$ & -70 & 817.5 & 255.1 & -70 & 113.1 & -90 \\
\hline YTX & -70 & 1141.6 & 1061.6 & -55 & 855.5 & -70 \\
\hline homo-YTX & -70 & 1155.6 & 1075.6 & -55 & 869.5 & -70 \\
\hline
\end{tabular}


A UHPLC system (1290 Infinity II, Agilent Technologies, Waldbronn, Germany) with a 0.3 min dwell time was coupled to a 6550 iFunnel QToF (Agilent Technologies, Santa Clara, CA, USA) equipped with a dual ESI source. This instrument was operated with a dual electrospray ion source with Agilent Jet Stream Technology ${ }^{\mathrm{TM}}$ in positive $\left(\mathrm{ESI}^{+}\right)$and negative (ESI-) ionization modes. Mass spectra were acquired over the scan range $\mathrm{m} / z, 100-1200$ with an acquisition rate of $0.5 \mathrm{~s}$. The parameters of the Jet Stream Technologies ${ }^{\mathrm{TM}}$ source in $\mathrm{ESI}^{+}$were: gas temperature $205{ }^{\circ} \mathrm{C}$, drying gas flow $16 \mathrm{~L} / \mathrm{min}$, nebulizer pressure $50 \mathrm{psig}$, sheath gas temperature $355^{\circ} \mathrm{C}$, sheath $12 \mathrm{~L} / \mathrm{min}$, capillary voltage $2 \mathrm{kV}$, fragmentor voltage, $200 \mathrm{~V}$. In ESI the parameters were as follows: gas temperature $290{ }^{\circ} \mathrm{C}$, drying gas flow $12 \mathrm{~L} / \mathrm{min}$, nebulizer pressure $50 \mathrm{psig}$, sheath gas temperature $355^{\circ} \mathrm{C}$, sheath $12 \mathrm{~L} / \mathrm{min}$, capillary voltage $-3.5 \mathrm{kV}$, fragmentor voltage, $180 \mathrm{~V}$. The instrument was mass calibrated in positive and negative ionization modes before each analysis, using the Agilent tuning mix. Additionally, all experiments were carried out using reference mass correction using purine $\left(\mathrm{m} / z 121.05087[\mathrm{M}+\mathrm{H}]^{+}\right.$; $\left.\mathrm{m} / \mathrm{z} 119.03632[\mathrm{M}-\mathrm{H}]^{-}\right)$and HP-921 = hexakis $(1 \mathrm{H}, 1 \mathrm{H}, 3 \mathrm{H}$-tetrafluoropropoxy) phosphazine $(\mathrm{m} / \mathrm{z}$ $\left.922.00979[\mathrm{M}+\mathrm{H}]^{+} ; \mathrm{m} / z 966.00072[\mathrm{M}+\mathrm{HCOO}]^{-}\right)$. The reference ions were infused constantly with an isocratic pump to a separate ESI sprayer in the dual spray source. MassHunter Acquisition B05.01 software was used to control the instrument and data were processed with MassHunter B07.00.

\section{System C: Orbitrap:}

Analyses were carried out on an Accela ${ }^{\mathrm{TM}}$ High Speed LC (dwell time $0.75 \mathrm{~min}$ ) coupled to an Exactive $^{\mathrm{TM}}$ mass spectrometer (ThermoFisher Scientific, Whaltham, MA, USA), equipped with an Orbitrap mass analyzer and a heated electrospray ionization probe (HESI-II). The instrument was operated and mass calibrated in positive and negative ionization modes as described previously [14]. "Balanced" automatic gain control (AGC) was used for all analyses, with a maximum injection time set to $50 \mathrm{~ms}$ across a scan range of $\mathrm{m} / \mathrm{z} 100$ - 1500. Data acquisition was carried out with Xcalibur software (ThermoFisher Scientific).

Optimal ion source and interface conditions consisted of a spray voltage of $3 \mathrm{kV}$ (positive mode) or $2.7 \mathrm{kV}$ (negative mode), sheath gas flow rate of $50\left(\mathrm{ESI}^{+}\right)$and $25(\mathrm{ESI})$, auxiliary gas flow rate of 10 , capillary temperature of $360^{\circ} \mathrm{C}$ and heater temperature of $250^{\circ} \mathrm{C}$. Acquisitions were made in full scan with high collision dissociation (HCD) using an energy of $60 \mathrm{eV}$. Full scan and HCD data were acquired at high (50000) and medium (10000) resolutions respectively. Alternative full scan and HCD data were obtained at a scan rate of $2 \mathrm{~Hz}$, resulting in an overall cycle time of ca. $1 \mathrm{~s}$.

\subsubsection{Liquid chromatography conditions}

Three different Kinetex stationary phases (C18, XB-C18 and Biphenyl from Phenomenex) of identical geometry and particle size have been initially evaluated (see supplementary material Table S1). The column finally selected was a Phenomenex Kinetex XB-C18 (100 x 2.1 mm; $2.6 \mu \mathrm{m})$.

The binary mobile phase consisted of (A) 100\% water and (B) $95 \%$ acetonitrile. All phases contained $2 \mathrm{mM}$ ammonium formate and $50 \mathrm{mM}$ formic acid. The final gradient selected after optimization of chromatographic separation used a flow-rate of $400 \mu \mathrm{L} / \mathrm{min}$, and acetonitrile in the organic component. The elution gradient rose from $5 \%$ to $50 \%$ of B in $3.6 \mathrm{~min}$, then $100 \% \mathrm{~B}$ was reached by 8.5 min. After $1.5 \mathrm{~min}$ of hold time at $100 \% \mathrm{~B}, 5 \% \mathrm{~B}$ was reached within $10 \mathrm{~s}$, followed by 5 min reequilibration of the column at $5 \% \mathrm{~B}$. The total chromatographic run time was $15 \mathrm{~min}$. For all experiments the column temperature was maintained at $40^{\circ} \mathrm{C}$ and injection volumes were $3 \mu \mathrm{L}$. This gradient was used to compare the chromatographic separation between columns in the triple quadrupole system and also to assess matrix effects in all three mass spectrometry systems listed above. 


\subsection{Sample preparation}

\subsubsection{Mussel, passive samplers and CRMs extraction protocol}

Mussel (Mytilus galloprovincialis) and blank HP-20 passive samplers (300 mg) used to prepare matrix-matched calibration solutions had been deployed over the same 1-week period at Villefranchesur-mer bay (France). Mussels were prepared according to the EURLMB SOP [38] by extracting $2 \mathrm{~g}$ of homogenized mussels with $2 \times 9 \mathrm{~mL}$ of $100 \% \mathrm{MeOH}$. After centrifugation, the supernatants were combined into a volumetric flask and the volume adjusted to $20 \mathrm{~mL}$ using $\mathrm{MeOH}$. Passive samplers were prepared and extracted as described [33]. SPATTs were prepared from HP20 resin (300 mg) contained between sheets of mesh that were hold together by embroidery rings. After retrieval, each SPATT was rinsed with deionized water, the resin transferred to an empty SPE cartridge and eluted with $15 \mathrm{~mL}$ of $\mathrm{MeOH}$. Since the procedure for the preparation of matrix-matched standard required diluting the matrix extract to $3 / 4$ of the original volume, initial blank extracts were concentrated to $4 / 3$ of the original volume under a gentle stream of nitrogen, to yield appropriate matrix concentration in the final matrix-matched solutions. A protocol adapted from McCarron et al [13] was used to extract CRMs samples. CRM material $(2 \mathrm{~g})$ was serially extracted four times with $5.5 \mathrm{~mL}$ of $\mathrm{MeOH}$. The supernatants were collected and brought to $25 \mathrm{~mL}$ into a volumetric flask.

\subsubsection{Matrix-matched calibration solutions for the evaluation of matrix effects}

Due to potential stability problems of AZAs, PnTX-E and PTX2 in acidic conditions [39-41] (and the acid present in the certified calibrant to enhance storage capacity of 13-desmeSPX-C), three initial toxin mixtures were prepared in methanol: (i) Mix-1 containing PTX2, AZA1 to 3, OA, DTX1 and 2, PnTX-E, YTX, homo-YTX and DA; (ii) Mix-2 containing 13-desmeSPX-C, GYM-A, PnTX-F, PnTX-G and DA and (iii) BTX1,2-mix with BTX1 and BTX2. These stock solutions were then serially diluted in $\mathrm{MeOH}$ using a Hamilton Microlab diluter-dispenser (Hamilton Company, Reno, NV). The samples from the serial dilution series were spiked into previously prepared and concentrated blank mussel and SPATT extracts (from section 2.3.1): firstly, aliquots of extract (225 $\mu \mathrm{L}$ ) were dispensed into HPLC vials, then $75 \mu \mathrm{L}$ of each dilution level solution was added. This operating procedure resulted in a consistent matrix concentration at each concentration level. Matrixfree samples were prepared similarly, using pure methanol instead of mussel or passive sampler extracts.

The calibration curves thus covered a range from approximately $0.07 \mathrm{ng} \mathrm{mL}^{-1}$ to $50 \mathrm{ng} \mathrm{mL}^{-1}$ for AZAs and okadaic acid groups, $0.04 \mathrm{ng} \mathrm{mL}^{-1}$ to $26 \mathrm{ng} \mathrm{mL}^{-1}$ for cyclic imines, $0.3 \mathrm{ng} \mathrm{mL}^{-1}$ to $220 \mathrm{ng} \mathrm{mL}^{-1}$ for YTXs, $1.5 \mathrm{ng} \mathrm{mL}^{-1}$ to $1070 \mathrm{ng} \mathrm{mL}^{-1}$ for DA, $11 \mathrm{ng} \mathrm{mL}^{-1}$ to $740 \mathrm{ng} \mathrm{mL}^{-1}$ for BTX1 and $25 \mathrm{ng} \mathrm{mL}^{-1}$ to $1620 \mathrm{ng} \mathrm{mL}^{-1}$ for BTX2. Based on triplicate injections of seven points methanol and matrix-matched calibration curves, mean slopes, intercept and correlation coefficients $\left(\mathrm{R}^{2}\right)$ were calculated by application of least squares adjustment without weighting.

Matrix effects were evaluated on the QqQ, the Q-ToF and on the Orbitrap using the Phenomenex Kinetex XB-C18 $(100 \times 2.1 \mathrm{~mm} ; 2.6 \mu \mathrm{m})$ column with the optimized gradient.

\subsection{Method performance characteristics}


To assess method performances and matrix effects, each concentration for each calibration curve was injected in triplicate, alternating between standards in methanol, standards in SPATT matrix and standards in mussel matrix. After the injection of each matrix-matched calibration curve, a check standard sample containing the monitored toxins was injected in-between two blank injections. This procedure led to injection sequences of approximately 100 injections. Drift correction, if necessary, was applied before any further data processing: evaluation of linearity, accuracy, matrix effects, etc. (supplementary material S1).

Mass-to-charge ratio on high resolution instruments and the corresponding standard deviations were calculated from triplicate injections of methanol, SPATT or mussel calibration solutions. Mass extraction was made with a mass accuracy window of $\pm 5 \mathrm{ppm}$. To avoid positive and negative errors cancelling each other out when calculating errors (ppm) [42], absolute values of the individual mass errors were used.

As there is not always sufficient noise to calculate signal-to-noise ratios in HRMS, detection limits (LoD) were determined with the ordinary least-squares regression data method [43, 44] using the lowest 3 points from the calibration curves (in $\mathrm{MeOH}$, SPATT and mussel extracts). The LoD was calculated as 3 times the standard deviation of the y-intercepts, over the slope of the calibration curve $[43,44]$.

To evaluate the accuracy of the method on all three systems (QqQ, Q-ToF and Orbitrap), certified reference materials containing targeted toxins at known concentrations were analyzed: CRM-ASPmus-d for DA; CRM-DSP-mus-c for OA, DTX1 and -2; CRM-AZA-mus-d for AZA-1, -2 and -3 and CRM-FDMT-1 for 13-desme-SPX-C and PTX2.

\subsection{Data treatment}

Statistical evaluations were carried out using SigmaPlot 12.5. Significance tests used to compare matrix effects between different conditions were a t-test, a Wilcoxon signed rank test and an ANOVA on ranks according to Friedman using repeated measures. Differences were considered significant at $p$ $<0.05$.

The Agilent Molecular Feature Extractor (MFE) algorithm was used to obtain the Total Compound Chromatogram of samples [45]. This algorithm is designed for use with full scan data and treats all of the mass spectral data as a three-dimensional array of retention time, $\mathrm{m} / \mathrm{z}$ and abundance values. At this stage, any point corresponding to persistent or slowly-changing background is removed from that array of values. Subsequently, the algorithm searches for ion traces (=Features) that have common elution profile, i.e. ion traces that elute at very nearly the same retention times. Those ion traces are then grouped into entities called Compounds regrouping all ion traces that are related, i.e. those that correspond to mass peaks in the same isotope cluster, or can be explained as being different adducts or charge states of the same entity. The results for each detected Compound are a mass spectrum containing the ions with the same elution time and explainable relationships, and an extracted compound chromatogram (ECC) computed using all of these related ion traces in the compound spectrum (and only those traces). Finally, all Compounds eluting at very nearly the same retention time are grouped into compound groups to facilitate data reduction. Indeed, the algorithm does not allow for regrouping of true fragments different from adducts or isotopic clusters, and thus two or more of the entities called Compounds from a same group may actually be derived from in-source fragmentation of a single molecule.

Non-targeted analysis of field samples often show more complex blanks as all ionisable compounds from the solvents and additives used in extraction, sample preparation and mobile phases, as well as ghost-peaks from previous injections, may appear in the mass analyzer. Thus, some samples were blank-subtracted post-acquisition for evaluation of data complexity. For this blank-subtraction, a 
database was constituted with all peaks that appeared in solvent blanks and HP20 (passive sampler = SPATT matrix) extraction blanks. When using the $M F E^{\mathrm{TM}}$ algorithm described above, an exclusion list may be added to exclude these compounds present in the blank from those extracted into total compound chromatograms (TCCs). Whenever blank subtraction was applied this is specifically mentioned in the result and discussion section.

\section{RESULTS AND DISCUSSION}

\subsection{Method Selection and Performance}

Initial chromatographic method development focused on achieving good separations within the OA group to avoid quantitation errors (different toxicity of OA and DTX2). During method development Kinetex C18, Kinetex XB-C18 and Kinetex Biphenyl columns $(100 \times 2.1 \mathrm{~mm} ; 2.6 \mu \mathrm{m})$ were compared (Supplementary material Table S1). Better resolutions between neighboring peaks (Rs $>2$ ) were obtained on Kinetex $\mathrm{C} 18$ and XB-C18 compared to the Kinetex Biphenyl (supplementary material Table S2). Of note AZA3 and PTX2 were resolved on the Kinetex XB-C18 column (Rs=6.9) but not on the Kinetex $\mathrm{C} 18$, probably due to the slightly higher polarity of the Kinetex XB-C18 column, as well as its different steric interactions [46].

A methanol-based mobile phase was also tested with the same gradient on the three columns. Methanol has a selectivity different to that of acetonitrile, and use of the same gradient led to more coelution between toxins, regardless of the column stationary phase, in particular the type of bonding (supplementary material Table S2). Therefore, the mobile phase with methanol was discarded for further experiments. However, it is noteworthy that better sensitivity was obtained for BTXs when using the methanol mobile phase, compared to the acetonitrile mobile phase (supplementary material Figure S1).

The column finally chosen was the Kinetex XB-C18, with resolutions of Rs=6.9 between PTX2 and AZA3, Rs=4.5 between OA and DTX2 and Rs=3.2 between YTX and OA. PnTX-F and PnTX-G were barely baseline resolved (Rs=2), but significant co-elution remained for BTX2 and AZA2 (Rs=1.1) in positive ionization, and for YTX and homo-YTX in negative ion mode (supplementary material Table S2 and Figure S2). We aimed to develop a relatively short method for a multiclass screening of phycotoxins. Figure 1 shows the LC separation of 29 different algal toxins using the optimized gradient. LRMS and HRMS approaches for multi-toxin determination were examined further using these conditions for a reduced set of toxins as certified calibration solutions were not available for all toxins. 


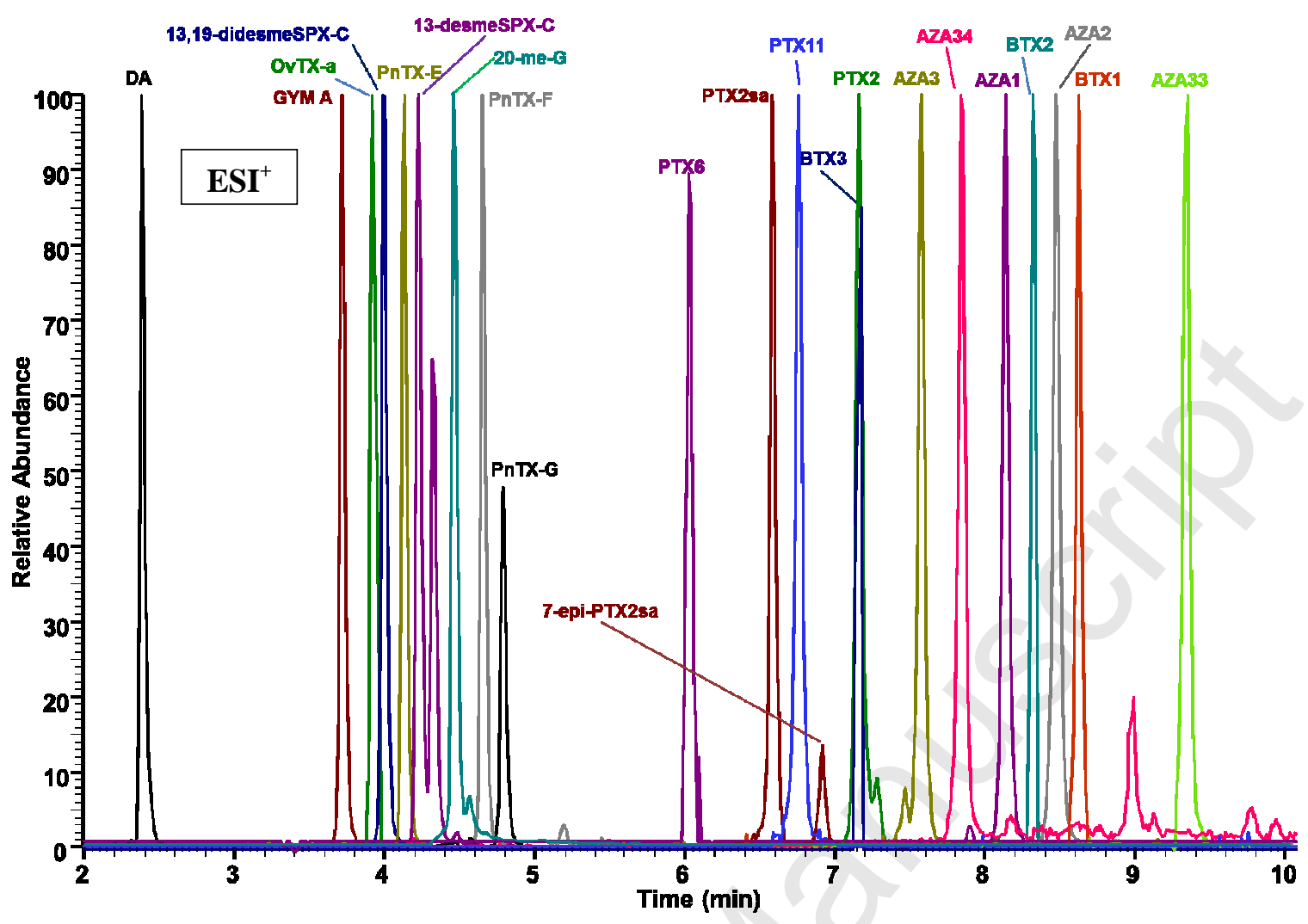

330

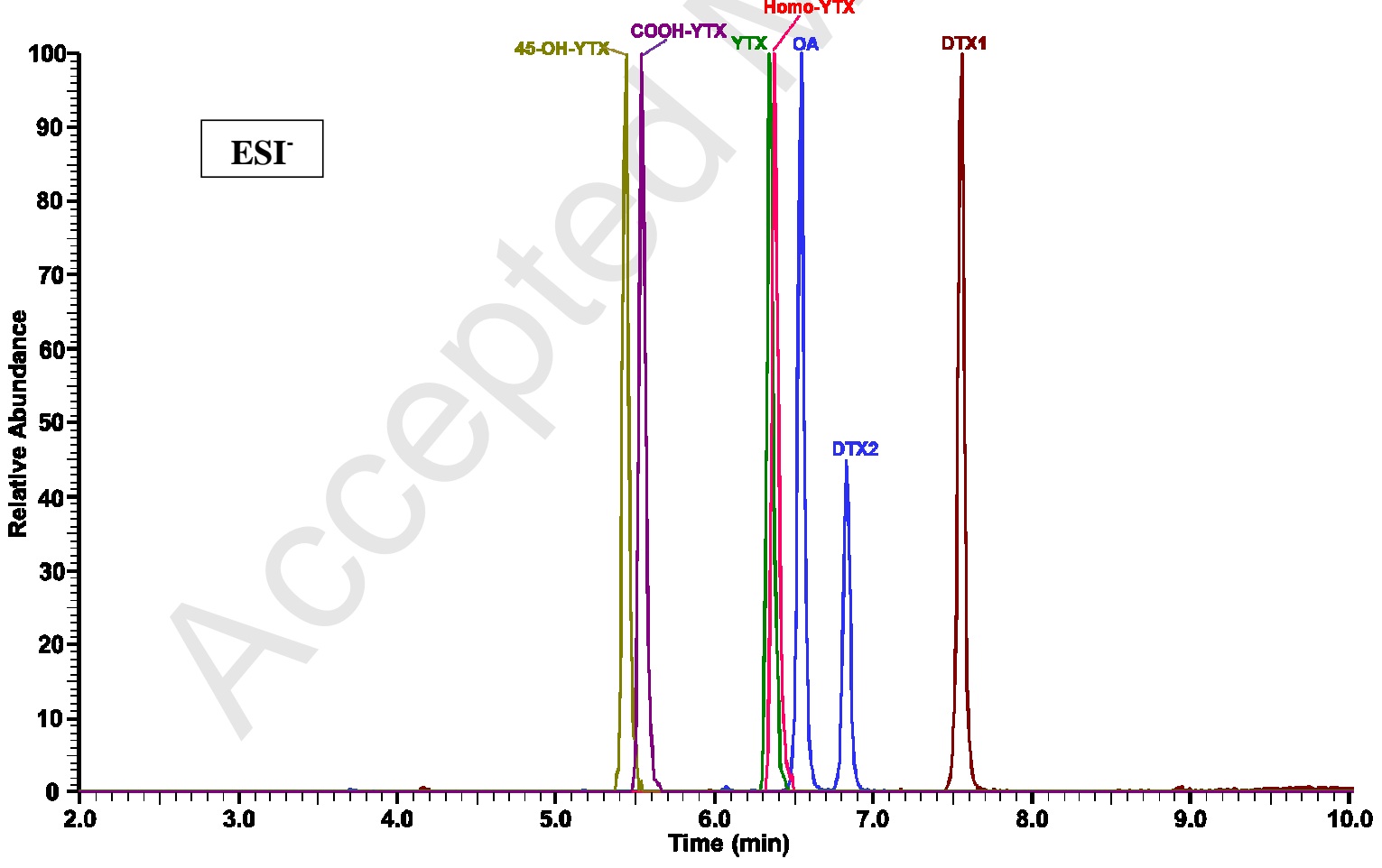

Figure 1: HPLC chromatogram in $\mathrm{ESI}^{+}$and $\mathrm{ESI}^{-}$, of the composite multi-toxin sample (section 2.1) acquired on the Orbitrap using the Phenomenex Kinetex XB-C18 (100 x $2.1 \mathrm{~mm}$; 2.6 $\mu \mathrm{m})$ with the optimized gradient (acetonitrile). 
There was good reproducibility of retention times throughout the entire gamut of injection sequences (101 injections) on all the instruments. Indeed, standard deviation for retention times were all below $0.16 \mathrm{~min}(\mathrm{n}=63)$ (Table 2). Some shifts in retention times were observed as could be expected between Systems A to $\mathrm{C}$ due to different delay volumes.

Table 2: Reproducibility of retention times $(\mathrm{RT} \pm \mathrm{SD})$ throughout a 24 h injection sequence $(\mathrm{n}=63)$ on all systems using Phenomenex Kinetex XB-C18 $(100 \times 2.1 ; 2.6 \mu \mathrm{m})$ and the optimized gradient.

Retention times \pm SD $(\min )$

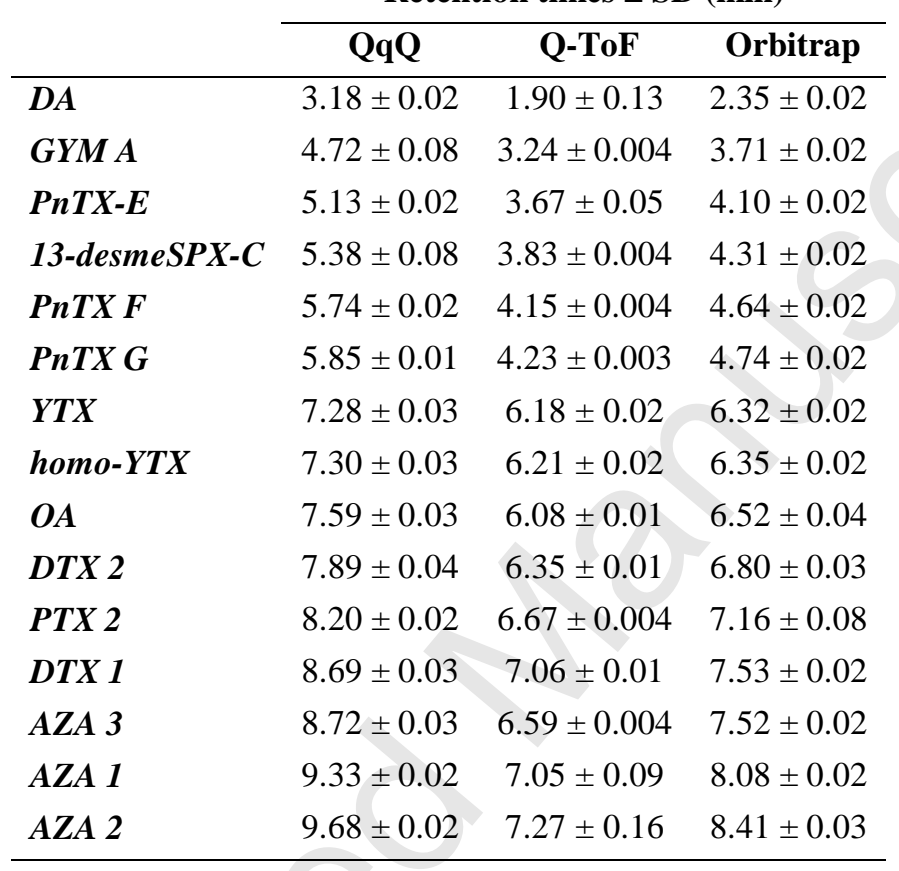

Mass accuracy measured for both instruments ranged between $0.31 \mathrm{ppm}$ to $3 \mathrm{ppm}$ in positive mode and between $0.39 \mathrm{ppm}$ to $3.42 \mathrm{ppm}$ in negative mode (see supplementary material Table S3). Under defined conditions, both instruments claim sub-ppm mass accuracy in full scan mode, and our study showed overall good mass accuracy $(<3.5 \mathrm{ppm})$. Furthermore, the highest mass errors were not observed for the same compounds (or ionization modes) on the two high resolution systems: on Q-ToF the highest mass errors were obtained in negative mode for YTX and homo-YTX, while on the Orbitrap the highest mass error was observed in positive mode for PTX2.

Standard deviations of the error of mass measurements ranged from 0.05 to $1.56 \mathrm{ppm}$ for the Q-ToF and, from $0.17 \mathrm{ppm}$ to $1.04 \mathrm{ppm}$ for the Orbitrap. Overall, standard deviations of the error were slightly lower on the Orbitrap $(0.46 \mathrm{ppm})$ compared to the Q-ToF $(0.58 \mathrm{ppm})$. These results showed a good stability in mass measurement for both instruments and were consistent with previously published mass accuracy data [14, 15]. For all toxins on all instruments, mean calibration curves obtained from triplicate injections of methanol, SPATT and mussel solutions showed good linearity $\left(\mathrm{R}^{2}>0.99\right)$ (see supplementary material Table $\left.\mathrm{S} 4\right)$.

\subsection{Evaluation of matrix effects}

\subsubsection{Mussel $v s$ passive sampler matrix effects}


The response including the matrix effect was expressed as a percentage of the response obtained for each compound in $\mathrm{MeOH}$ and determined by comparison of the mean slope of methanol calibration curves $(n=3)$ to those of matrix-matched calibration curves $(n=3)$. Responses $>100 \%$ correspond to ion enhancement while responses $<100 \%$ reflect ion suppression. For all three systems, passive sampler matrix quantitatively led to less matrix effects than mussel matrix (Figure 2). The largest matrix effects were observed for PTX2, OA, DTX1 and DTX2, and in all these cases, ion enhancement was observed.

Fewer toxins were affected by matrix effects on the Q-ToF (statistically significantly). However, matrix effects on the Q-ToF were among the highest of the three systems for PTX2 $(+53 \%)$, OA $(+111 \%)$, DTX1 $(+222 \%)$, DTX2 $(+119 \%)$ and DA $(+49 \%)$. Still, in these chromatographic conditions, no ion suppression higher than $16 \%$ was observed for any toxin on the Q-ToF, regardless of the matrix. For the other two systems (QqQ and Orbitrap), a greater number of toxins were affected by matrix effects (statistically significantly). On the QqQ ion enhancement was also observed for DA $(+14 \%)$, PTX2 (+2\%), OA (+62\%), DTX1 (+77\%) and DTX2 (+51\%), while AZA1,-2 and -3 suffered respectively from $28 \%, 31 \%$ and $27 \%$ ion suppression. These were overall among the highest ion suppression phenomena observed. On the Orbitrap, ion enhancement was again observed for PTX2 $(+65 \%)$, DTX1 (+31\%) and DTX2 (+20\%) and, ion suppression for GYM A $(-30 \%)$, PnTX-F $(-24 \%)$, PnTX-G (-22\%), YTX (-23\%) and homo-YTX (-23\%). Since the most important factor in non-target screening is detectability, a system and chromatographic conditions should be chosen to avoid or minimize ion suppression. In the conditions tested, the Q-ToF gave least ion suppression. This finding should certainly be taken with caution as different matrices and chromatographic conditions should also be evaluated on all systems before generalizing this conclusion.

Due to unfavorably high detection limits, matrix effects and detection limits for BTX1 and BTX2 were not fully evaluated. On the triple quadrupole, while negligible ion suppression $(<10 \%)$ was observed with the passive sampler matrix for BTX1 and BTX2, the mussel matrix gave the highest ion suppression (-13\% for BTX1 and -29\% for BTX2) (data not shown).

Although the causes of matrix effects are not fully understood, it is a common assumption that such effects may originate from competition between an analyte and co-eluting matrix components for the available charges inside the ionization source [17-20]. In fact, all three instruments used in this study had electrospray ionization sources. Therefore, it is not surprising that similar matrix effects were observed on all three systems for those toxins susceptible to the largest matrix effects (PTX2, OA, DTX1 and -2); the only difference being the degree of suppression or enhancement.

Also, regardless of the analytical system used, mussel matrix almost always led to quantitatively more matrix effects than passive sampler matrix. This was expected as mussels are biological samples, consequently containing multiple exogenous and endogenous compounds that may have further undergone biotransformation. The SPATT extract was obtained from HP20 resin exposed to the Mediterranean Sea (1-week deployment). Notably, the resin had already been pre-extracted with $\mathrm{MeOH}$ for activation prior to deployment. Hence, most of the technical by-products that may still be present in technical polymeric resin had been washed out. Additionally, different extraction protocols for mussels and SPATTs yielded extract with different matrix concentrations (solvent-to-sample ratios of 10 for mussel and 33.3 for SPATT). Therefore, the SPATT sample was presumed to contain significantly less matrix components and the observation of lower matrix effects from SPATT than from mussel samples may be attributed, at least in part, to this difference. Previous studies have demonstrated the ability of passive samplers to accumulate biotoxins quite effectively [30, 33, 47]. Since our study clearly shows that passive sampler matrix causes much less matrix effects, we would recommend this technique for non-targeted studies as a complementary tool useful to toxin discovery or dereplication. 

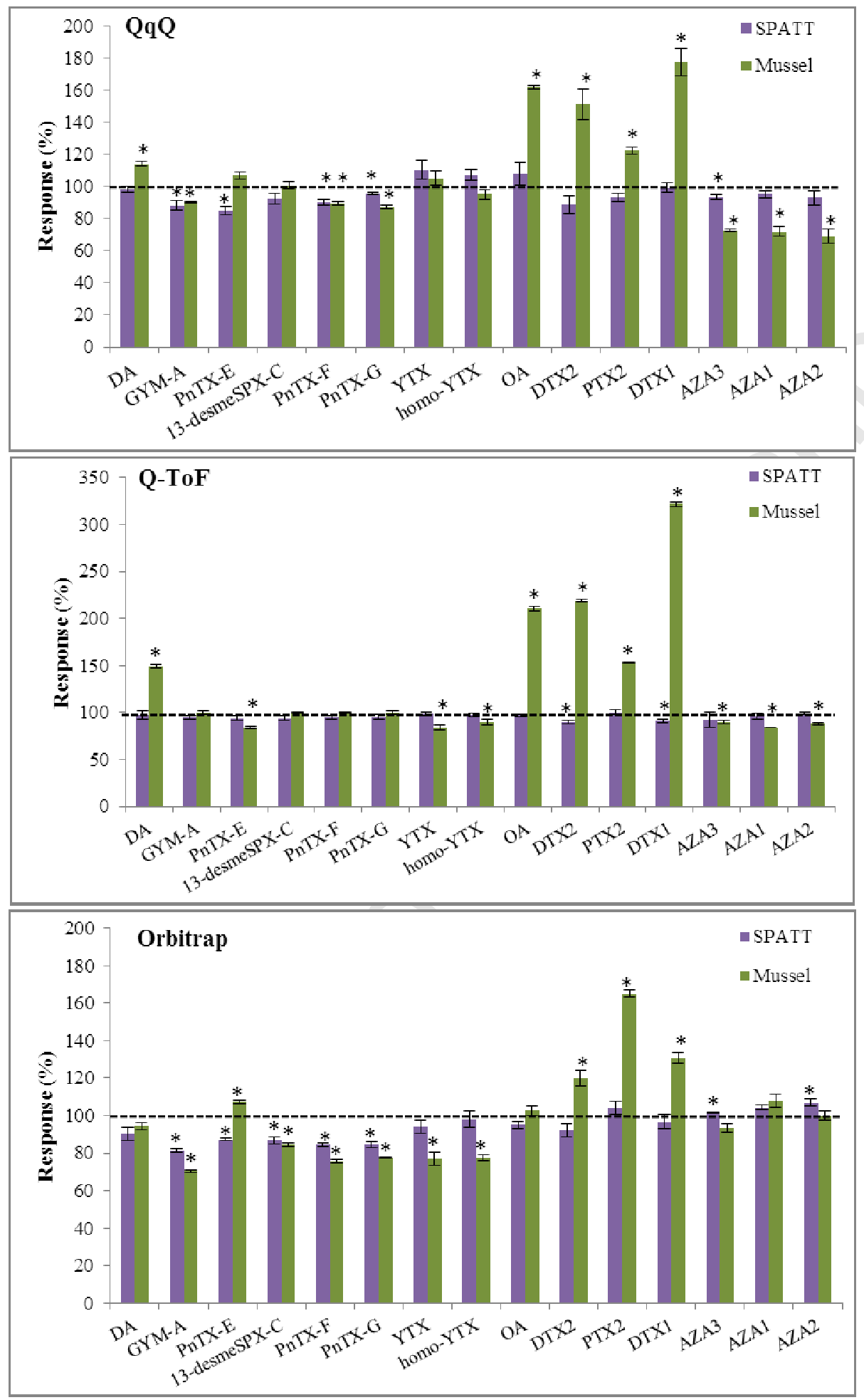

Figure 2: SPATT and mussel matrix effects (\% response compared to response in $\mathrm{MeOH} \pm \% \mathrm{RSD}$, $\mathrm{n}=3$ ) for various toxins using three instrument systems. Asterisk $\left(^{*}\right)$ indicates that response for matrix is statistically significantly different $(\mathrm{p}<0.05)$ than response for methanol $(\mathrm{t}$-test). Nota bene: For PTX2, the ammonium adduct was used for the quantitative evaluation of matrix effects on all instruments. The other toxins were quantitated using the $[\mathrm{M}+\mathrm{H}]^{+}$or the $[\mathrm{M}-\mathrm{H}]^{-}$. 


\subsection{Parameters affecting matrix effects}

\subsubsection{Ion chosen for quantitation}

Characteristic ions for PTX2 in positive electrospray ionization are: $\mathrm{m} / \mathrm{z} 876.5\left(\left[\mathrm{M}+\mathrm{NH}_{4}\right]^{+}\right)$and $\mathrm{m} / \mathrm{z}$ $881.4\left([\mathrm{M}+\mathrm{Na}]^{+}\right)$, and the proportions can vary depending on instrument and method set-up. On all the HRMS systems the sodium adduct gave a higher response than the ammonium adduct; however, the relative abundances of sodium $v s$. ammonium ions initially present in the ion source at the time of desorption/ionization, and the generally higher stability of sodium adducts in the ion source will certainly influence this competition. It was therefore necessary to assess what importance the quantifier ion could have on matrix effects. This comparison was undertaken on both high resolution mass spectrometers (QToF, and Orbitrap) using methanol and matrix-matched calibration solutions. On both instruments, either no matrix effect (SPATT) or ion enhancement (mussel) was observed when the ammonium adduct was used for quantitation. However, mostly ion suppression was observed when using the sodium adduct (Figure 3). Differences in matrix effects obtained with the sodium or ammonium adducts were statistically significant according to a t-test $(\mathrm{p}<0.05)$, except for SPATT samples on the Orbitrap. The sodium adduct not being subject to changes in volatility, ion suppression is the more likely matrix effect, due to the presence of high concentrations of nonvolatile compounds in the spray inside the source [22]. Furthermore, a reduced evaporation rate of the most volatile species present including ammonia may also lead to increased ammonium concentration thereby causing the enhancement of ammonium adducts.

The use of sodium adducts is not recommended for quantitation both due to the suppression observed and because of their inherent resistance to fragmentation for structure confirmation purposes [29]. However, in a full scan screening approach using HRMS, this ion still provides some benefits. The sodium adduct could be used for better sensitivity and as a confirmatory ion, however, caution should be taken when interpreting ion abundance ratios.

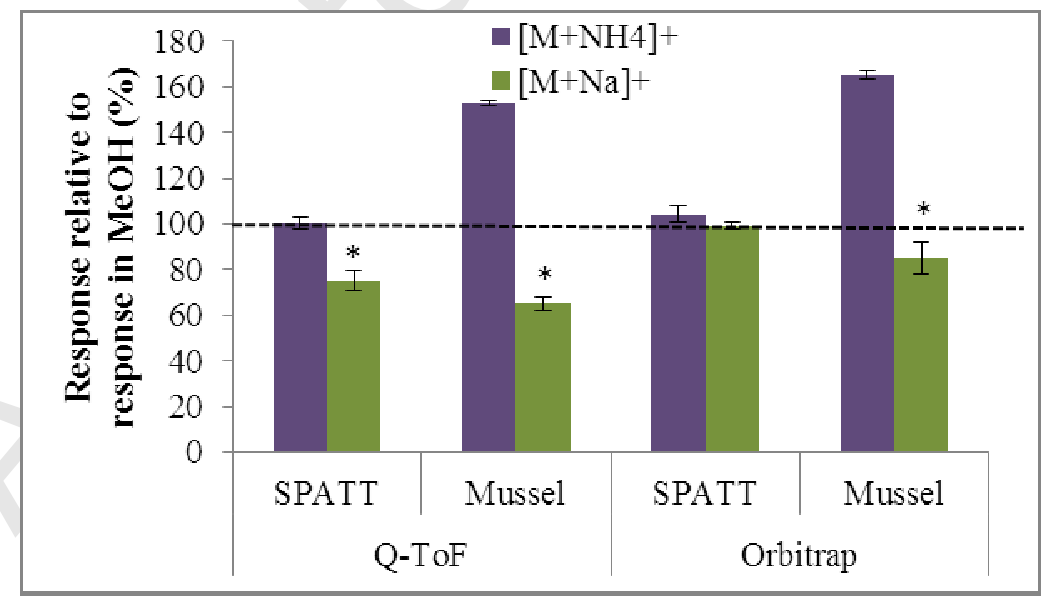

Figure 3: Matrix effects (\% response compared to response in $\mathrm{MeOH} \pm \% \mathrm{RSD}, \mathrm{n}=3$ ) for PTX2 in SPATT and mussel samples using ammonium $\left(\left[\mathrm{M}+\mathrm{NH}_{4}\right]^{+}\right)$and sodium $\left([\mathrm{M}+\mathrm{Na}]^{+}\right)$adducts. Nota bene: the absolute response of the sodium adduct is higher than that of the ammonium adduct, the response shown here is relative to the response in $\mathrm{MeOH}$. An asterisk (*) indicates that response of adducts for this matrix-toxin combination is statistically significantly different $(p<0.05)$ from the response in methanol (individual t-test for each matrix-instrument combination). 
453

454

455

456

457

458

459

460

461

462

463

464

A simple way of reducing matrix effects is to reduce the amount of matrix entering the system, either by use of smaller injection volumes or by diluting the sample [11, 13]. On the Q-ToF matrix effects were compared using matrix-matched calibration solutions prepared either with crude or 10-fold diluted extracts. In crude SPATT extracts, only ion suppression had been observed, and dilution reduced matrix effects to a negligible level $(<10 \%)$ (Figure 4$)$. These differences were, however, only statistically significant for OA $(\mathrm{p}=0.007)$, DTX1 $(\mathrm{p}=0.028)$ and DTX2 $(\mathrm{p}=0.003)$, where the largest matrix effects had been observed for crude extracts. For mussel samples, the reduction in matrix effects was significant for 7 out of 11 compounds evaluated. Again, matrix effects were less significant for passive samplers. While dilution is beneficial in terms of matrix effects its application must be considered in relation to dilution of toxin signal response.
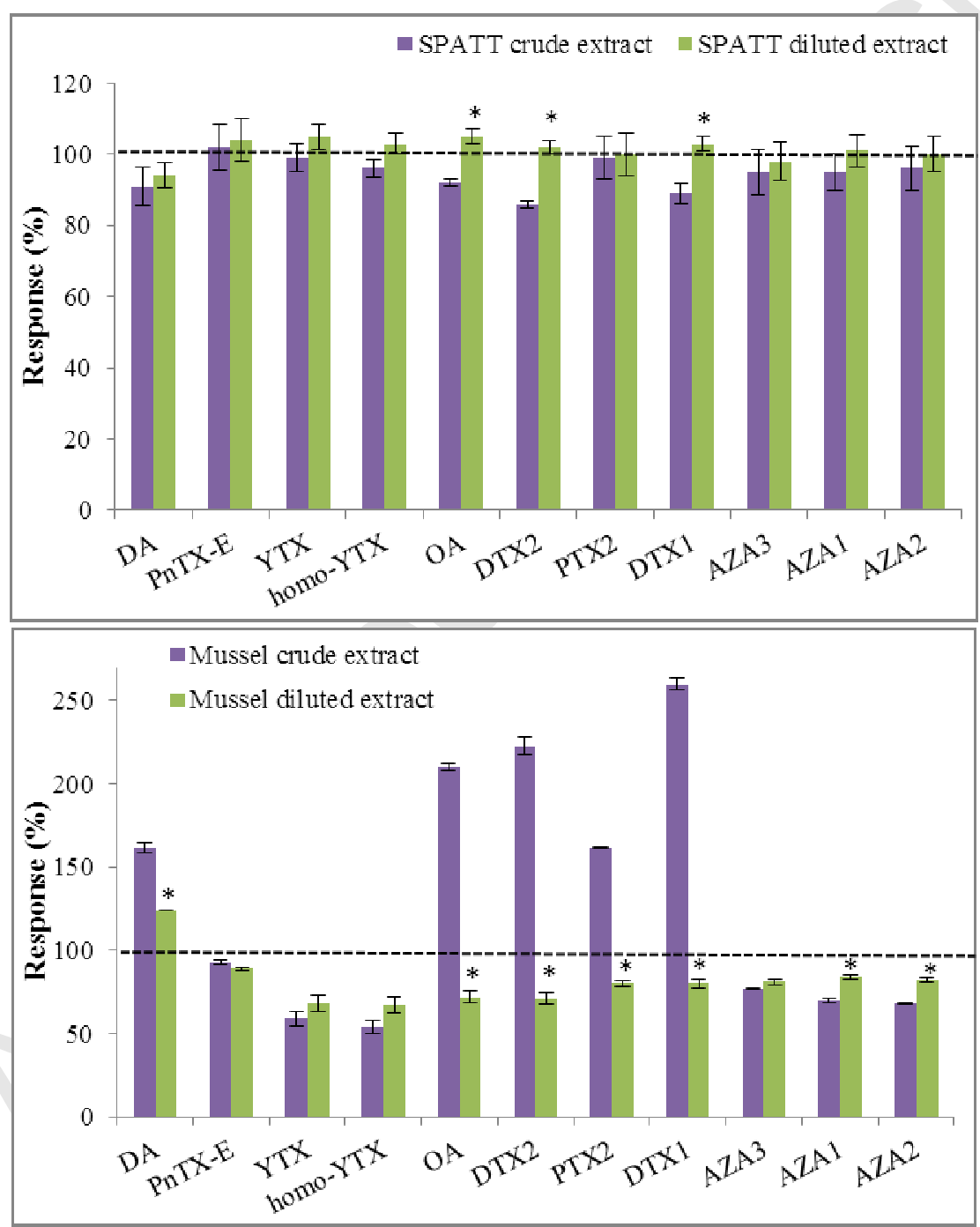

Figure 4: Matrix effects on Q-ToF (\% response compared to response in $\mathrm{MeOH} \pm \% \mathrm{RSD}, \mathrm{n}=3$ ) obtained with calibration solutions prepared using crude or diluted SPATT (top) and mussel (bottom) extracts. Asterisk $(*)$ indicates that response of diluted extracts for this matrix-toxin combination is statistically significantly different $(\mathrm{p}<0.05)$ from the response in crude extracts (individual t-test for each matrix-toxin combination). 


\subsection{Low resolution $v s$ high resolution mass spectrometry}

472

Since matrix effects were observed on both low and high resolution analytical systems (section 3.2.1), analysis of high resolution mass spectra of our targeted toxins was undertaken. PTX2 in positive ionization and DTX1 in negative ionization were the toxins with the highest matrix effects, irrespective of the instrument used. High resolution full scan spectra at the retention times of these toxins showed more co-eluting compounds in mussel matrix than in passive sampler matrix (Figure 5). As already discussed passive sampler extracts potentially contained less matrix components overall. Consequently, less co-elution would be expected during the analysis of these extracts. Despite matrix co-elution with DTX1 and PTX2, no interfering ions were observed when zooming in on the targeted $\mathrm{m} / \mathrm{z}$ values of interest. These results suggest that mass interference played no role in the matrix effects observed. While HRMS could neither reduce or remove matrix effects in this study, it does offer the ability to perform quantitative screening of known toxins as efficiently as low resolution MS/MS. In addition, HRMS also facilitates retrospective screening of any additional analogues or metabolites and enables untargeted screening via database screening and metabolomics software.

To illustrate the advantages of using full scan HRMS for untargeted analysis, chromatograms were plotted for all compounds potentially present in a SPATT and mussel sample taken at the same location and time (Figure 6). Both chromatograms exhibited a high number of compounds. The mussel sample contained significantly more compounds than the SPATT sample, either over the whole chromatogram or over the time span over which toxins eluted (Figure 6). For clarity, Figure 6 shows only a single sample each of SPATT and mussel extracts, however, statistical assessment of non-targeted data was carried out on triplicate injections. In this particular case, the chromatograms were blank-subtracted after acquisition (see section 2.5). The blank subtraction removed on average 197 and 210 compounds from the SPATT and mussel chromatograms, respectively. After blank-subtraction, SPATT and mussel extracts contained 814 and 4562 compounds respectively. Moreover, the absolute abundances of compounds in the passive sampler were much lower (Figure 6). Therefore, the data complexity is more than five-fold reduced by using passive samplers rather than complex biological models, such as mussels. Interestingly, approximately half of the compounds found in the passive samplers were also present in the mussel sample. This reflects well the fact that metabolites from micro-organisms (including algal metabolites) are efficiently adsorbed passively on the SPATT samplers in addition to being actively ingested as particulate matter by mussels. The fact that on average 363 compounds were unique to extracts of the passive sampler also means that the passive samplers may capture compounds from the dissolved phase which are not available to mussels due to inefficient absorption via the gills. Such compounds may be derived from algal blooms that occurred elsewhere with dissolved metabolites being advected with currents to the sampling area. They may also be from cryptic organisms that are present in the sampling area, e.g. as benthic or epiphytic micro-algae, but are not available to filtration feeding mussels as the compounds are in the dissolved and not in the particulate phase. If this were indeed the case, then, the accumulation of dissolved compounds in the passive samplers may be considered to be an "over-estimation" of the risk that such compounds pose to the consumer of shellfish. However, in the case of non-targeted, exploratory analysis of waters from a coastal area, any overestimation could be considered to err on the side of caution. Inversely, the metabolism pathways that algal toxins undergo in shellfish may be considered a complexity that merits further exploration. 
514

515

516

517

518

519

520

521

522

523

524

525

526

527

528

529

530

531

532

533

534

535

536

537

538

539

540
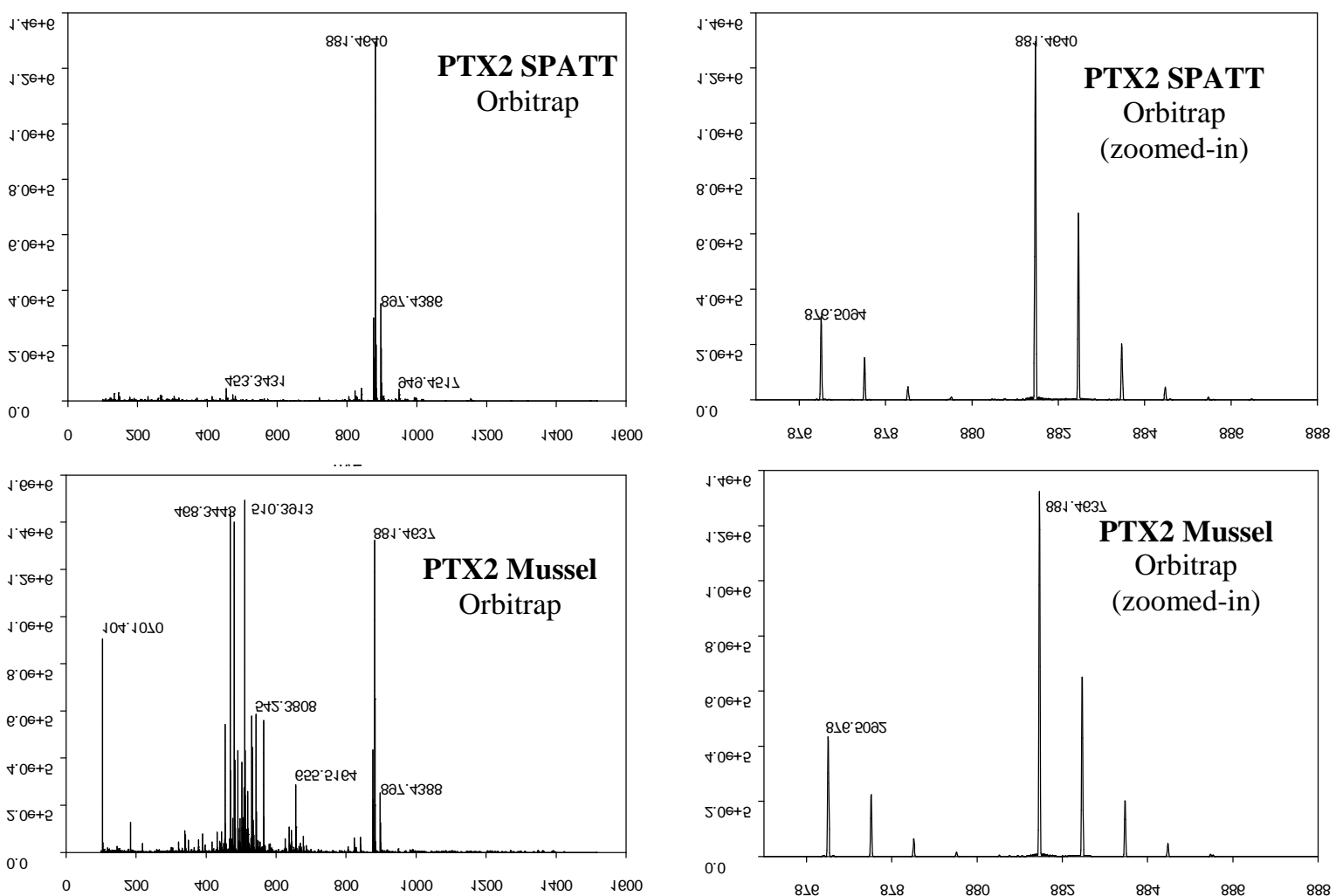

ine
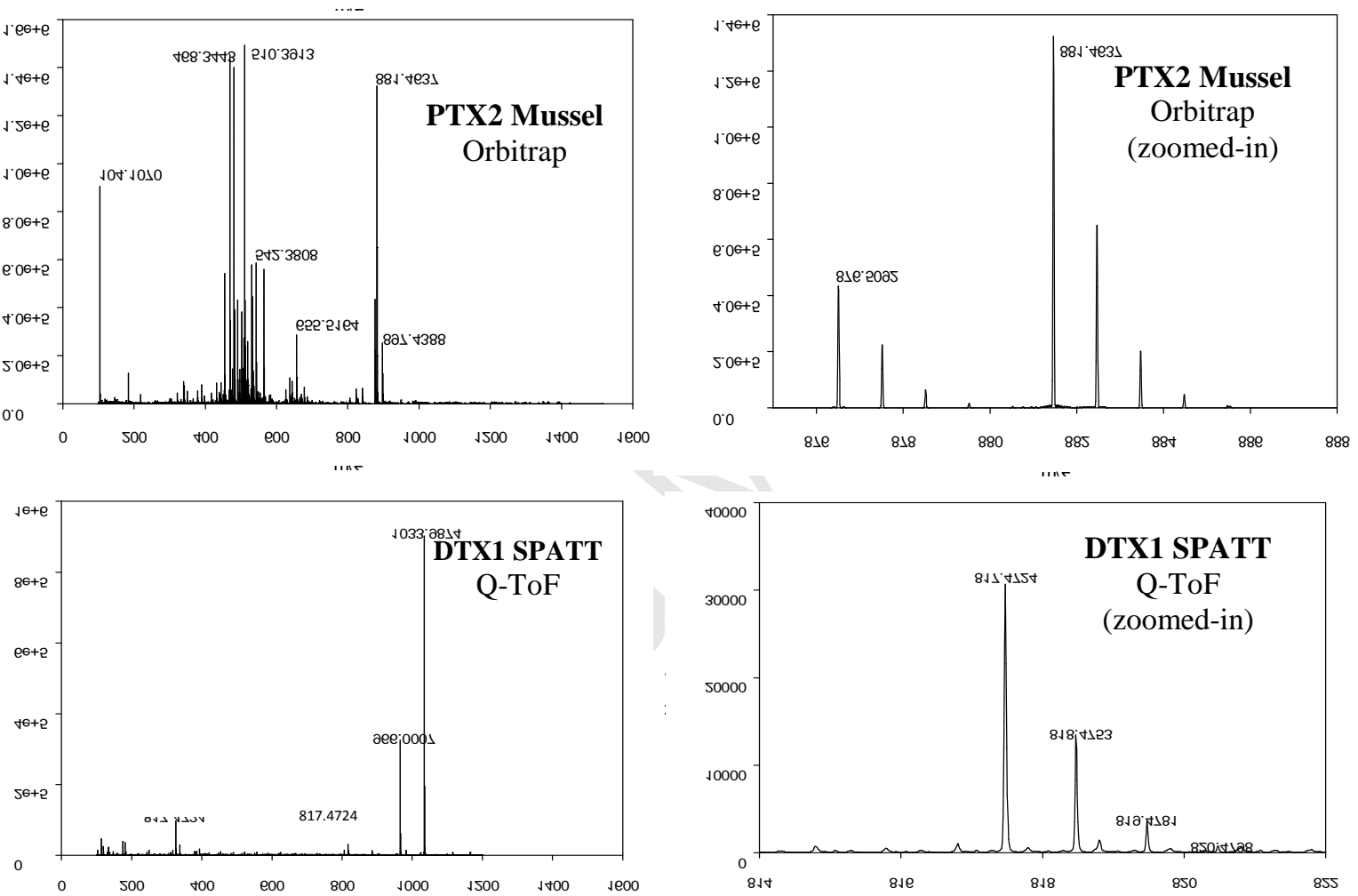

une
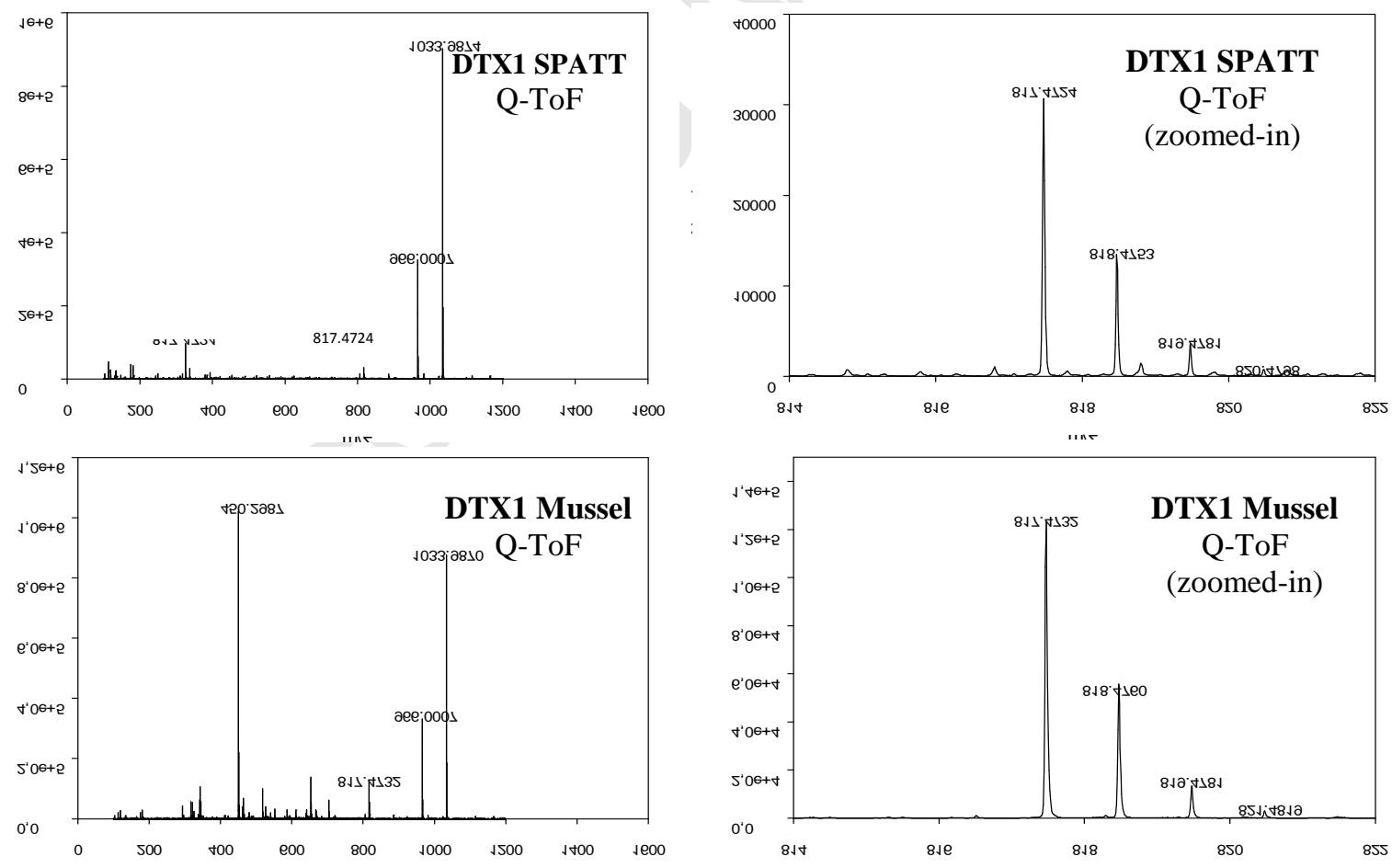

Figure 5: PTX2 (Orbitrap) and DTX1 (Q-ToF) high resolution mass spectra on the apex of the peak for SPATT and mussel extracts. Images on the left-hand side represent the whole spectrum while images on the right-hand side are zoomed-in on the $\mathrm{m} / \mathrm{z}$ of interest, showing no interfering masses. Nota bene: the ion $\mathrm{m} / \mathrm{z} 966.0007$ in Q-ToF spectra is from the continuously infused reference mass. 


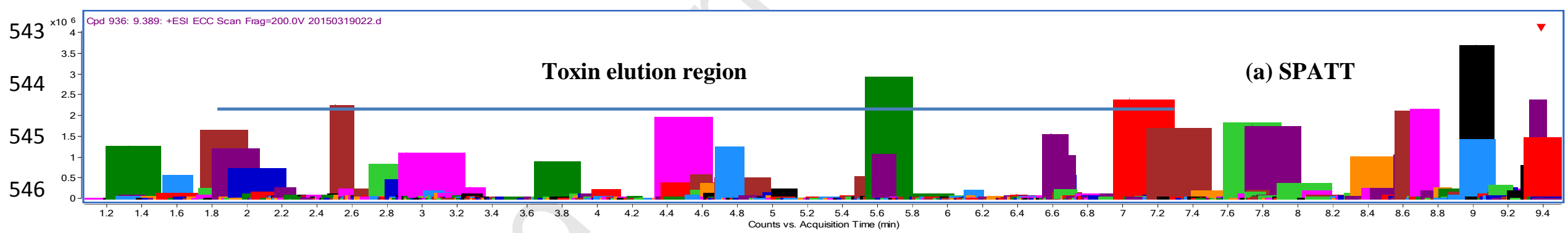

$547_{\times 10} 7$ Cpd 4251: 9.391: +ESI ECC Scan Frag=200.0V 20150319033.d

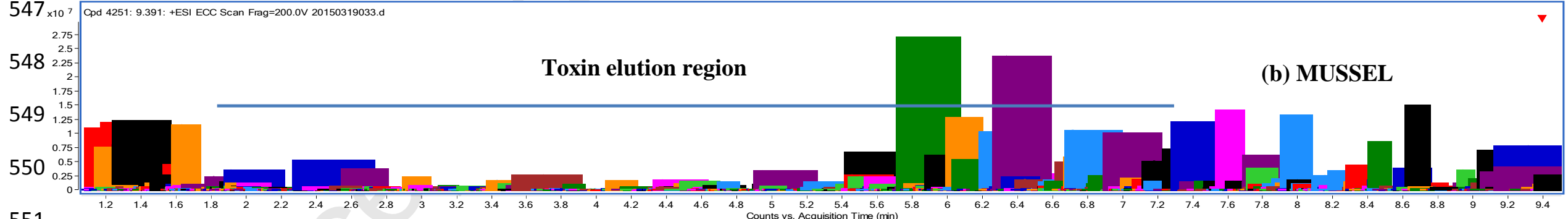

Figure 6: Total compound chromatogram (TCC) of the spiked blank SPATT sample (a) and blank mussel sample (b), both deployed at Villefranche during the same week. TCCs were obtained using the "Find by Molecular Feature"-algorithm and show the complexity of each sample (all compounds from 1 to $9.5 \mathrm{~min}$ ). The TCC was blank-subtracted, i.e. compounds appearing in blank solvent or blank HP20 matrix extracts were removed. There were 936 compounds present in the SPATT sample and 4251 compounds present in mussel sample. The number of compounds in the region where toxins eluted (1.8 $7.3 \mathrm{~min}$ ) was 619 and 2542 for the SPATT and the mussel sample, respectively. Nota bene: the scale of the TCC for the SPATT sample is ca. eight times lower than that of the mussel sample, reflecting a reduced total abundance of matrix compounds in SPATT matrix. 


\subsection{Accuracy and detection limits}

560

561

562

563

564

565

566

567

568

569

570

571

572

573

574

575

576

577

578

579

580

581

582

583

584

585

586

587

588

589

Mussel tissue CRMs were analyzed to assess the accuracy of the method. For many toxins recoveries were acceptable, ranged from $80 \%$ to $120 \%$ on low and high resolution systems, and were comparable between the different instruments (Table 3). This was not the case for DA on the QToF (121\%), PTX2 on all instruments (123-135\%), YTX on the Orbitrap and Q-ToF (52\% and 51\%, respectively) and for OA (64\%) and AZA3 (72\%) on the Q-ToF. The CRM results were not entirely consistent with the matrix effects observed in the evaluation work for the same toxins (Figure 2), however, the type and magnitude of observed matrix effects were generally consistent. Recoveries for the CRMs were calculated based on methanol calibration solutions. As the CRM matrices were different from the mussel sample matrix used in the matrix effect evaluation study it is not surprising that the CRM recoveries were not entirely consistent with the observed matrix effects.

Table 3: Recoveries from CRMs (\% \pm RSD; $n=3$ ): CRM-ASP-mus-d (DA), CRM-DSP-mus-c (OA, DTX1 and -2), CRM-AZA-mus-d (AZA1, -2 and -3) and CRM-FDMT-1 (13-desmeSPX-C, PTX2).

(*) The authentic NRC CRM certificates should be referred to for the original certified values.

\begin{tabular}{|c|c|c|c|c|}
\hline \multirow[b]{2}{*}{ Toxin } & \multirow[b]{2}{*}{$\begin{array}{c}\text { Target value* } \\
\left(\mu g g^{-1}\right)\end{array}$} & \multicolumn{3}{|c|}{ Recoveries (\%) } \\
\hline & & QqQ & Q-ToF & Orbitrap \\
\hline$D A$ & $49 \pm 3$ & $104 \pm 2$ & $121 \pm 1$ & $117 \pm 1$ \\
\hline 13-desmeSPX-C* & $2.7 \pm 0.14$ & $92 \pm 6$ & $90 \pm 4$ & $88 \pm 1$ \\
\hline$A Z A 1$ & $1.16 \pm 0.10$ & $83 \pm 7$ & $88 \pm 2$ & $95 \pm 2$ \\
\hline$A Z A 2$ & $0.27 \pm 0.02$ & $98 \pm 1$ & $106 \pm 1$ & $112 \pm 2$ \\
\hline AZA3 & $0.21 \pm 0.02$ & $90 \pm 5$ & $72 \pm 4$ & $109 \pm 3$ \\
\hline PTX2 & $0.68 \pm 0.06$ & $135 \pm 3$ & $134 \pm 4$ & $123 \pm 11$ \\
\hline$O A$ & $1.05 \pm 0.8$ & $97 \pm 3$ & $64 \pm 25$ & $97 \pm 15$ \\
\hline DTXI & $1.05 \pm 0.02$ & $94 \pm 5$ & $85 \pm 46$ & $120 \pm 4$ \\
\hline DTX2 & $0.85 \pm 0.02$ & $121 \pm 5$ & $76 \pm 24$ & $112 \pm 2$ \\
\hline YTX & $2.57 \pm 0.25$ & $81 \pm 8$ & $51 \pm 40$ & $52 \pm 4$ \\
\hline
\end{tabular}

Instrumental limits of detection (LoDs) were equivalent between instruments, with the exception of YTX and homo-YTX on the QqQ which gave the lowest detection limits, and OA where the QqQ gave the highest detection limit (Table 4). LoDs varied between analytes but also between matrices. Indeed, lower LoDs were expected for PTX2 in mussel compared to passive sampler, as the mussel matrix caused ion enhancement for PTX2. This was not the case, perhaps reflecting the high level of variability previously associated with this toxin $[6,13]$. With regard to regulatory levels for toxins, satisfactory detection limits were obtained on both low and high resolution mass spectrometers for methanol, mussel and SPATT matrices. Even for the OA-group which had relatively high LoDs, the sum of detection limits results in a limit of quantitation (LoQ) of $\sim 51 \mu \mathrm{g}$ OA-equivalent $\mathrm{kg}^{-1}$ shellfish matrix. To obtain this value the individual LoDs were weighted by the relative toxicity of the analogues and summed up. The toxicity of DTX1 is the same as that of OA while the toxicity of DTX2 is only half that of OA. Subsequently, the sum of weighted LoDs was multiplied by 3 to obtain the LoQ. This is approximately three times below the regulatory limit for this group ( $160 \mu \mathrm{g} \mathrm{kg}^{-1} \mathrm{OA}$ equivalents). In general the good detection limits obtained with HRMS for the other toxins illustrated the capacity for quantitative screening of toxins in comparison with more conventional LRMS 
approaches. The significant added advantage of HRMS is the ability to perform full scan and MS/MS acquisitions simultaneously $[15,16]$, and thus enable retrospectively evaluation of data.

\section{CONCLUSIONS}

LRMS and HRMS were compared for quantitative and qualitative screening on toxins in mussels and passive samplers. Matrix effects were similar on all instruments (e.g. ion enhancement for PTX2, OA, DTX1 and DTX2), and generally were consistent with previously published results. There were significantly less matrix effects associated with passive samplers than mussels, regardless of the toxin or the instrument used. While high resolution would not be expected to alleviate matrix effects, it was demonstrated that the matrix effect issue for toxin measurement by LC-MS was not mass interference during ion detection. Acquisition of high resolution mass spectra enabled visualization of co-eluting compounds and clarified the absence of interfering masses.

Accuracy was good with all analytical systems. An approach such as matrix-matched calibration can be used to correct matrix effects, but to do so effectively would require a blank matrix which affects ionization to the same extent as the matrix of samples of interest. Sample dilution significantly reduced matrix effects in mussel matrix, while it made the issue effectively negligible for the passive sampler matrix. For PTX2, quantitation using the ammonium-adduct led to ion enhancement compared to the sodium-adduct; however, the ammonium adduct yields more fragments for comprehensive confirmation.

This study assessed parameters involved in quantitative analysis of biotoxins in mussels and passive samplers, using LRMS and HRMS. The minimal matrix effects associated with passive samplers, as well as the reduced data complexity, means that passive sampling in combination with HRMS is a technique with great utility for non-targeted screening of algal toxins in the marine environment. 
614

615

616

Table 4: Detection limits (LoDs) in ng analyte $\mathrm{mL}^{-1}$ injected solution ( $3 \mu \mathrm{L}$ injected of either methanol, SPATT or mussel matrix solutions) and in $\mu \mathrm{g} \mathrm{kg}^{-1}$ (mussel matrix) on QqQ, Q-ToF and Orbitrap.

Detection limits $\left(\mathbf{n g ~ m L ^ { - 1 } )}\right.$

\begin{tabular}{|c|c|c|c|c|c|c|c|c|c|c|c|c|}
\hline \multirow{3}{*}{ Toxin } & \multicolumn{9}{|c|}{ Detection limits $\left(\mathrm{ng} \mathrm{mL} \mathrm{m}^{-1}\right)$} & \multirow{2}{*}{\multicolumn{3}{|c|}{$\frac{\text { Detection limits }\left(\mu \mathrm{g} \mathrm{kg}^{-1}\right)}{\text { Mussel }}$}} \\
\hline & \multicolumn{3}{|c|}{ QqQ } & \multicolumn{2}{|r|}{ Q-ToF } & & \multicolumn{3}{|c|}{ Orbitrap } & & & \\
\hline & $\mathrm{MeOH}$ & Spatt & Mussel & $\mathrm{MeOH}$ & Spatt & Mussel & $\mathrm{MeOH}$ & Spatt & Mussel & QqQ & Q-ToF & Orbitrap \\
\hline$D A$ & 3.07 & 1.17 & 2.87 & 1.27 & 1.06 & 2.53 & 1.60 & 1.64 & 2.41 & 28.7 & 25.3 & 24.1 \\
\hline$G Y M-A$ & 0.10 & 0.01 & 0.16 & 0.003 & 0.03 & 0.09 & 0.01 & 0.01 & 0.09 & 1.61 & 0.90 & 0.87 \\
\hline$P n T X-E$ & 0.03 & 0.06 & 0.05 & 0.07 & 0.01 & 0.05 & 0.01 & 0.08 & 0.01 & 0.46 & 0.47 & 0.09 \\
\hline 13-desmeSPX-C & 0.04 & 0.06 & 0.41 & 0.02 & 0.05 & 0.02 & 0.02 & 0.02 & 0.03 & 4.13 & 0.15 & 0.30 \\
\hline$P n T X-F$ & 0.02 & 0.05 & 0.01 & 0.02 & 0.02 & 0.03 & 0.01 & 001 & 0.03 & 0.07 & 0.31 & 0.31 \\
\hline$P n T X-G$ & 0.01 & 0.01 & 0.02 & 0.02 & 0.02 & 0.01 & 0.02 & 0.01 & 0.03 & 0.22 & 0.09 & 0.29 \\
\hline YTX & 0.34 & 0.71 & 1.24 & 1.82 & 1.51 & 2.15 & 1.83 & 1.71 & 3.35 & 12.4 & 21.5 & 33.5 \\
\hline homo-YTX & 0.29 & 0.51 & 1.18 & 0.69 & 1.76 & 1.74 & 1.58 & 2.60 & 0.54 & 11.8 & 17.4 & 5.39 \\
\hline$O A$ & 2.29 & 1.88 & 1.43 & 0.23 & 0.65 & 0.17 & 0.15 & 0.34 & 0.56 & 14.3 & 1.67 & 5.6 \\
\hline DTX2 & 0.47 & 0.48 & 0.20 & 0.12 & 0.08 & 0.19 & 0.41 & 0.42 & 0.30 & 1.95 & 1.94 & 3.02 \\
\hline$P T X 2$ & 0.10 & 0.55 & 0.10 & 0.06 & 0.07 & 0.17 & 0.08 & 0.06 & 0.12 & 1.00 & 1.66 & 1.20 \\
\hline DTX1 & 0.12 & 0.03 & 0.19 & 0.15 & 0.08 & 0.33 & 0.44 & 0.11 & 0.49 & 1.94 & 3.29 & 4.91 \\
\hline$A Z A 3$ & 0.03 & 0.04 & 0.05 & 0.05 & 0.03 & 0.05 & 0.01 & 0.03 & 0.06 & 0.45 & 0.52 & 0.64 \\
\hline$A Z A 1$ & 0.09 & 0.06 & 0.14 & 0.06 & 0.03 & 0.04 & 0.02 & 0.01 & 0.02 & 1.36 & 0.40 & 0.20 \\
\hline$A Z A 2$ & 0.18 & 0.08 & 0.21 & 0.06 & 0.07 & 0.11 & 0.01 & 0.04 & 0.24 & 2.14 & 1.07 & 2.38 \\
\hline
\end{tabular}




\section{CONFLICT OF INTEREST}

The authors declare no competing financial interest.

\section{AKNOWLEDGMENTS}

This study was carried out under the Coselmar project supported by Ifremer and Nantes University and co-funded by the Regional Council of the "Pays de la Loire". The authors thank Thomas Glauner, Maxime Grives, John Lee and Thierry Faye of Agilent Technologies for their collaboration on the Agilent Q-ToF technology and software. The authors would also like to acknowledge Jean-Philippe Antignac (Laberca-ONIRIS, Nantes, France) for valuable discussions. We also thank all the members of the Laboratoire Phycotoxines at the Atlantic Centre of Ifremer and the Biotoxin Metrology team at the National Research Council of Halifax, for their help and advice during this study.

\section{REFERENCES}

[1] G.P. Rossini, P. Hess, Phycotoxins: chemistry, mechanisms of action and shellfish poisoning, Exs, 100 (2010) 65-122.

[2] P. Hess, B. Grune, D.B. Anderson, T. Aune, L.M. Botana, P. Caricato, H.P. van Egmond, M. Halder, S. Hall, J.F. Lawrence, C. Moffat, R. Poletti, J. Richmond, G.P. Rossini, C. Seamer, J.S. Vilageliu, Three Rs approaches in marine biotoxin testing - The report and recommendations of a joint ECVAM/DG SANCO workshop (ECVAM workshop 55), ATLA-Altern. Lab. Anim., 34 (2006) 193224.

[3] EURL, EU Reference Laboratory Marine Biotoxins, web-site, last accessed on 21 February 2013: http://www.aesan.msssi.gob.es/en/CRLMB/web/procedimientos_crlmb/crlmb_standard_operating_pro cedures.shtml, in, 2011.

[4] A. Brana-Magdalena, J. Manuel Leao-Martins, T. Glauner, A. Gago-Martinez, Infra laboratory Validation of a Fast and Sensitive UHPLC/MS/MS Method with Fast Polarity Switching for the Analysis of Lipophilic Shellfish Toxins, Journal of Aoac International, 97 (2014) 285-292.

[5] E. Fux, D. McMillan, R. Bire, P. Hess, Development of an ultra-performance liquid chromatography-mass spectrometry method for the detection of lipophilic marine toxins, Journal of chromatography. A, 1157 (2007) 273-280.

[6] H.J. van den Top, A. Gerssen, P. McCarron, H.P. van Egmond, Quantitative determination of marine lipophilic toxins in mussels, oysters and cockles using liquid chromatography-mass spectrometry: inter-laboratory validation study, Food Additives and Contaminants Part a-Chemistry Analysis Control Exposure \& Risk Assessment, 28 (2011) 1745-1757.

[7] A. These, C. Klemm, I. Nausch, S. Uhlig, Results of a European interlaboratory method validation study for the quantitative determination of lipophilic marine biotoxins in raw and cooked shellfish based on high-performance liquid chromatography-tandem mass spectrometry. Part I: collaborative study, Analytical and bioanalytical chemistry, 399 (2011) 1245-1256.

[8] P. McNabb, A.I. Selwood, P.T. Holland, Multiresidue method for determination of algal toxins in shellfish: Single-laboratory validation and interlaboratory study, Journal of Aoac International, 88 (2005) 761-772.

[9] M.A. Quilliam, P. Hess, C. Dell'Aversano, Recent developments in the analysis of phycotoxins by liquid chromatography-mass spectrometry, in, 2001, pp. 383-391.

[10] H.Y. Wu, M.M. Guo, Z.J. Tan, H.Y. Cheng, Z.X. Li, Y.X. Zhai, Liquid chromatography quadrupole linear ion trap mass spectrometry for multiclass screening and identification of lipophilic marine biotoxins in bivalve mollusks, Journal of Chromatography A, 1358 (2014) 172-180.

[11] P. McCarron, E. Wright, M.A. Quilliam, Liquid Chromatography/Mass Spectrometry of Domoic Acid and Lipophilic Shellfish Toxins with Selected Reaction Monitoring and Optional Confirmation by Library Searching of Product Ion Spectra, Journal of Aoac International, 97 (2014) 316-324. 
[12] P. McCarron, S.D. Giddings, K.L. Reeves, P. Hess, M.A. Quilliam, A mussel (Mytilus edulis) tissue certified reference material for the marine biotoxins azaspiracids, Analytical and bioanalytical chemistry, 407 (2015) 2985-2996.

[13] P. McCarron, S.D. Giddings, M.A. Quilliam, A mussel tissue certified reference material for multiple phycotoxins. Part 2: liquid chromatography-mass spectrometry, sample extraction and quantitation procedures, Analytical and bioanalytical chemistry, 400 (2011) 835-846.

[14] P. Blay, J.P. Hui, J. Chang, J.E. Melanson, Screening for multiple classes of marine biotoxins by liquid chromatography-high-resolution mass spectrometry, Analytical and bioanalytical chemistry, 400 (2011) 577-585.

[15] A. Domenech, N. Cortes-Francisco, O. Palacios, J.M. Franco, P. Riobo, J.J. Llerena, S. Vichi, J. Caixach, Determination of lipophilic marine toxins in mussels. Quantification and confirmation criteria using high resolution mass spectrometry, Journal of Chromatography A, 1328 (2014) 16-25.

[16] G. Orellana, J. Vanden Bussche, L. Van Meulebroek, M. Vandegehuchte, C. Janssen, L. Vanhaecke, Validation of a confirmatory method for lipophilic marine toxins in shellfish using UHPLC-HR-Orbitrap MS, Analytical and bioanalytical chemistry, 406 (2014) 5303-5312.

[17] B.K. Matuszewski, M.L. Constanzer, C.M. Chavez-Eng, Strategies for the assessment of matrix effect in quantitative bioanalytical methods based on HPLC-MS/MS, Analytical chemistry, 75 (2003) 3019-3030.

[18] J.P. Antignac, K. de Wasch, F. Monteau, H. De Brabander, F. Andre, B. Le Bizec, The ion suppression phenomenon in liquid chromatography-mass spectrometry and its consequences in the field of residue, Analytica chimica acta, 529 (2005) 129-136.

[19] R. King, R. Bonfiglio, C. Fernandez-Metzler, C. Miller-Stein, T. Olah, Mechanistic investigation of ionization suppression in electrospray ionization, Journal of the American Society for Mass Spectrometry, 11 (2000) 942-950.

[20] H. Trufelli, P. Palma, G. Famiglini, A. Cappiello, AN OVERVIEW OF MATRIX EFFECTS IN LIQUID CHROMATOGRAPHY-MASS SPECTROMETRY, Mass spectrometry reviews, 30 (2011) 491-509.

[21] T. Benijts, R. Dams, W. Lambert, A. De Leenheer, Countering matrix effects in environmental liquid chromatography-electrospray ionization tandem mass spectrometry water analysis for endocrine disrupting chemicals, Journal of Chromatography A, 1029 (2004) 153-159.

[22] F. Gosetti, E. Mazzucco, D. Zampieri, M.C. Gennaro, Signal suppression/enhancement in highperformance liquid chromatography tandem mass spectrometry, Journal of Chromatography A, 1217 (2010) 3929-3937.

[23] A. Gerssen, M.A. McElhinney, P.P.J. Mulder, R. Bire, P. Hess, J. de Boer, Solid phase extraction for removal of matrix effects in lipophilic marine toxin analysis by liquid chromatography-tandem mass spectrometry, Analytical and bioanalytical chemistry, 394 (2009) 1213-1226.

[24] J. Kilcoyne, E. Fux, Strategies for the elimination of matrix effects in the liquid chromatography tandem mass spectrometry analysis of the lipophilic toxins okadaic acid and azaspiracid-1 in molluscan shellfish, Journal of Chromatography A, 1217 (2010) 7123-7130.

[25] E. Fux, D. Rode, R. Bire, P. Hess, Approaches to the evaluation of matrix effects in the liquid chromatography-mass spectrometry (LC-MS) analysis of three regulated lipophilic toxin groups in mussel matrix (Mytilus edulis), Food Additives and Contaminants Part a-Chemistry Analysis Control Exposure \& Risk Assessment, 25 (2008) 1024-1032.

[26] S. Ito, K. Tsukada, Matrix effect and correction by standard addition in quantitative liquid chromatographic-mass spectrometric analysis of diarrhetic shellfish poisoning toxins, Journal of Chromatography A, 943 (2002) 39-46.

[27] A. Furey, J. Crowley, B. Hamilton, M. Lehane, K.J. James, Strategies to avoid the misidentification of anatoxin-a using mass spectrometry in the forensic investigation of acute neurotoxic poisoning, Journal of Chromatography A, 1082 (2005) 91-97.

[28] F. Mondeguer, J.P. Antignac, Y. Guitton, F. Monteau, S. LeBorgne, P. Hess, Nouvelle stratégie de caractérisation non ciblée de type métabolomique au service de l'identification de composés bioactifs accumulés dans les mollusques bivalves., Spectra Analyse, 284 (2012) 24-33.

[29] R.B. Cole, J.-C. Tabet, J.-C. Blais, Stabilities of fast atom bombardment desorbed alkali metal adducts of pyrimidine molecules, International Journal of Mass Spectrometry and Ion Processes, 98 (1990) 269-283. 
[30] L. MacKenzie, V. Beuzenberg, P. Holland, P. McNabb, A. Selwood, Solid phase adsorption toxin tracking (SPATT): a new monitoring tool that simulates the biotoxin contamination of filter feeding bivalves, Toxicon : official journal of the International Society on Toxinology, 44 (2004) 901-918.

[31] E. Fux, R. Bire, P. Hess, Comparative accumulation and composition of lipophilic marine biotoxins in passive samplers and in mussels (M. edulis) on the West Coast of Ireland, Harmful Algae, 8 (2009) 523-537.

[32] M. McCarthy, F.N.A.M. van Pelt, V. Bane, J. O'Halloran, A. Furey, Application of passive (SPATT) and active sampling methods in the profiling and monitoring of marine biotoxins, Toxicon : official journal of the International Society on Toxinology, 89 (2014) 77-86.

[33] Z. Zendong, C. Herrenknecht, E. Abadie, C. Brissard, C. Tixier, F. Mondeguer, V. Sechet, Z. Amzil, P. Hess, Extended evaluation of polymeric and lipophilic sorbents for passive sampling of marine toxins, Toxicon : official journal of the International Society on Toxinology, 91 (2014) 57-68. [34] J.-P. Lacaze, New trends in Marine Freshwater Toxins, Nova Science Publishers, Inc, 2012. [35] M. Garcia-Altares, A. Casanova, V. Bane, J. Diogene, A. Furey, P. de la Iglesia, Confirmation of Pinnatoxins and Spirolides in Shellfish and Passive Samplers from Catalonia (Spain) by Liquid Chromatography Coupled with Triple Quadrupole and High-Resolution Hybrid Tandem Mass Spectrometry, Marine drugs, 12 (2014) 3706-3732.

[36] G. Pizarro, A. Morono, B. Paz, J.M. Franco, Y. Pazos, B. Reguera, Evaluation of passive samplers as a monitoring tool for early warning of dinophysis toxins in shellfish, Marine drugs, 11 (2013) 3823-3845.

[37] C. Brissard, F. Herve, M. Sibat, V. Sechet, P. Hess, Z. Amzil, C. Herrenknecht, Characterization of ovatoxin-h, a new ovatoxin analog, and evaluation of chromatographic columns for ovatoxin analysis and purification, Journal of Chromatography A, 1388 (2015) 87-101.

[38] EU-RL-MB, EU-Harmonised Standard Operating Procedure for determination of Lipophilic marine biotoxins in molluscs by LC-MS/MS, in, EU-RL-MB, 2011, pp. 31.

[39] T. Suzuki, V. Beuzenberg, L. Mackenzie, M.A. Quilliam, Liquid chromatography-mass spectrometry of spiroketal stereoisomers of pectenotoxins and the analysis of novel pectenotoxin isomers in the toxic dinoflagellate Dinophysis acuta from New Zealand, Journal of Chromatography A, 992 (2003) 141-150.

[40] C. Alfonso, N. Rehmann, P. Hess, A. Alfonso, C.B. Wandscheer, M. Abuin, C. Vale, P. Otero, M.R. Vieytes, L.M. Botana, Evaluation of Various $\mathrm{pH}$ and Temperature Conditions on the Stability of Azaspiracids and Their Importance in Preparative Isolation and Toxicological Studies, Analytical chemistry, 80 (2008) 9672-9680.

[41] A.I. Selwood, C.O. Miles, A.L. Wilkins, R. van Ginkel, R. Munday, F. Rise, P. McNabb, Isolation, structural determination and acute toxicity of pinnatoxins E, F and G, Journal of agricultural and food chemistry, 58 (2010) 6532-6542.

[42] A.G. Brenton, A.R. Godfrey, Accurate Mass Measurement: Terminology and Treatment of Data, Journal of the American Society for Mass Spectrometry, 21 (2010) 1821-1835.

[43] J. Vial, A. Jardy, Experimental comparison of the different approaches to estimate LOD and LOQ of an HPLC method, Analytical chemistry, 71 (1999) 2672-2677.

[44] M.M. Sanagi, S.L. Ling, Z. Nasir, D. Hermawan, W.A.W. Ibrahim, A. Abu Naim, Comparison of Signal-to-Noise, Blank Determination, and Linear Regression Methods for the Estimation of Detection and Quantification Limits for Volatile Organic Compounds by Gas Chromatography, Journal of Aoac International, 92 (2009) 1833-1838.

[45] Agilent, Agilent Metabolomics Workflow Rev. B, http://www.chem.agilent.com/Library/usermanuals/Public/5990-

7067EN_MetabolomicsDiscoveryWorkflow_Guide_B.pdf, in, website, 29 Oct 2012, last accessed 24/06/2015.

[46] S.C. Moldoveanu, V. David, Chapter 6 - Stationary Phases and Their Performance, in: S.C.M. David (Ed.) Essentials in Modern HPLC Separations, Elsevier, 2013, pp. 191-362.

[47] E. Fux, C. Marcaillou, F. Mondeguer, R. Bire, P. Hess, Field and mesocosm trials on passive sampling for the study of adsorption and desorption behaviour of lipophilic toxins with a focus on OA and DTX1, Harmful Algae, 7 (2008) 574-583. 
772 [48] Agilent, Agilent All Ions MS/MS workflow guide,

773 https://www.chem.agilent.com/Library/usermanuals/Public/5991-

774 1994EN_All_Ions_MSMS_Guide.pdf, (website, 07 May 2013, last accessed 25/06/2015). 